\title{
P05 imaging beamline at PETRA III: first results
}

Imke Greving, Fabian Wilde, Malte Ogurreck, Julia Herzen, Jörg U. Hammel, et al.

Imke Greving, Fabian Wilde, Malte Ogurreck, Julia Herzen, Jörg U. Hammel, Alexander Hipp, Frank Friedrich, Lars Lottermoser, Thomas Dose, Hilmar Burmester, Martin Müller, Felix Beckmann, "P05 imaging beamline at PETRA III: first results," Proc. SPIE 9212, Developments in X-Ray Tomography IX, 921200 (12 September 2014); doi: 10.1117/12.2061768

Event: SPIE Optical Engineering + Applications, 2014, San Diego, California, United States 


\title{
P05 Imaging Beamline at Petra III - First Results
}

\author{
Imke Greving $^{a,}{ }^{*}$, Fabian Wilde ${ }^{a}$, Malte Ogurreck ${ }^{a}$, Julia Herzen ${ }^{a, b}$, Jörg U. Hammel ${ }^{a}$, \\ Alexander Hipp ${ }^{a}$, Frank Friedrich ${ }^{c}$, Lars Lottermoser ${ }^{a}$, Thomas Dose ${ }^{a}$, Hilmar Burmester ${ }^{a}$, \\ Martin Müller $^{a}$ and Felix Beckmann ${ }^{a}$ \\ ${ }^{a}$ Helmholtz Zentrum Geesthacht, Max-Plank-Str.1, 21502 Geesthacht, Germany \\ ${ }^{b}$ Technical University of Munich, Department of Physics (E17), Boltzmannstr. 11, 85748 \\ Garching, Germany \\ ${ }^{c}$ University of Hamburg, Biocenter Grindel \& Zoological Museum, \\ Martin-Luther-King-Platz 3, 20146 Hamburg, Germany
}

\begin{abstract}
The imaging beamline (IBL/P05) operated by Helmholtz Zentrum Geesthacht (HZG) at the DESY PETRA III storage ring consists of two experimental stations: A micro tomography and a nano tomography end station. Here an overview of the experimental setups and the data acquisition will be given. In addition some first results out of the wide range of applications using the micro tomography station at P05 will be shown. Furthermore, we present first results of the nano tomography end station. These were obtained with an $\mathrm{x}$-ray microscopy setup, which currently operates at energies of 17.4 and $30 \mathrm{keV}$ using polymer compound refractive lenses (CRLs) and rolled prism lenses. Taken together these results clearly show the high potential of the newly built imaging beamline IBL.
\end{abstract}

Keywords: Tomography, imaging, beamline, lenses, CRL

\section{INTRODUCTION}

X-ray computed tomography has become a vital tool for material sciences, biology, geology as well as medical sciences, in particular since the data acquisition time could be reduced significantly thanks to the high flux at $3^{\text {rd }}$ generation synchrotron sources. This method allows for three-dimensional visualization of a sample without having to cut or slice the sample. Visual inspection yields important information about the inner structure of the sample e.g. potential defects in newly developed materials. Apart from that it is also possible to obtain quantitative information from the tomograms, like pore size distributions, density fluctuations or other material specific parameters. Synchrotron radiation based tomography has several advantages compared to lab based sources: The low beam divergency and high monochromaticity reduces artefacts like beam hardening sufficiently and allows for a higher density resolution compared to lab based sources. Additionally the high flux at $3^{\text {rd }}$ generation Synchrotron sources reduces the acquisition time significantly and makes in particular in situ experiments and time resolved measurements possible. Resolutions in the range from a few micrometer down to about $<100$ nanometer can be reached and are ideal for a wide range of materials.

The high photon flux provided by PETRA III is well suited for imaging applications achieving high density and spatial resolutions. Furthermore, the high coherence at PETRA III is highly suited for phase contrast imaging techniques. Therefore, an imaging beamline has been set up at the storage ring which is particularly suited for materials science (e.g. quantitative analysis of pores, cracks, alloying processes), biology and geology (e.g. plants, insects, sediments) and medicine (e.g. implant materials, structures of bones, tissues, and teeth). In this article the technical setup of the imaging beamline IBL (P05) will be described alongside with first results and applications.

\footnotetext{
*E-mail: Imke.Greving@hzg.de, Telephone: +49 (0) 4089985304
} 


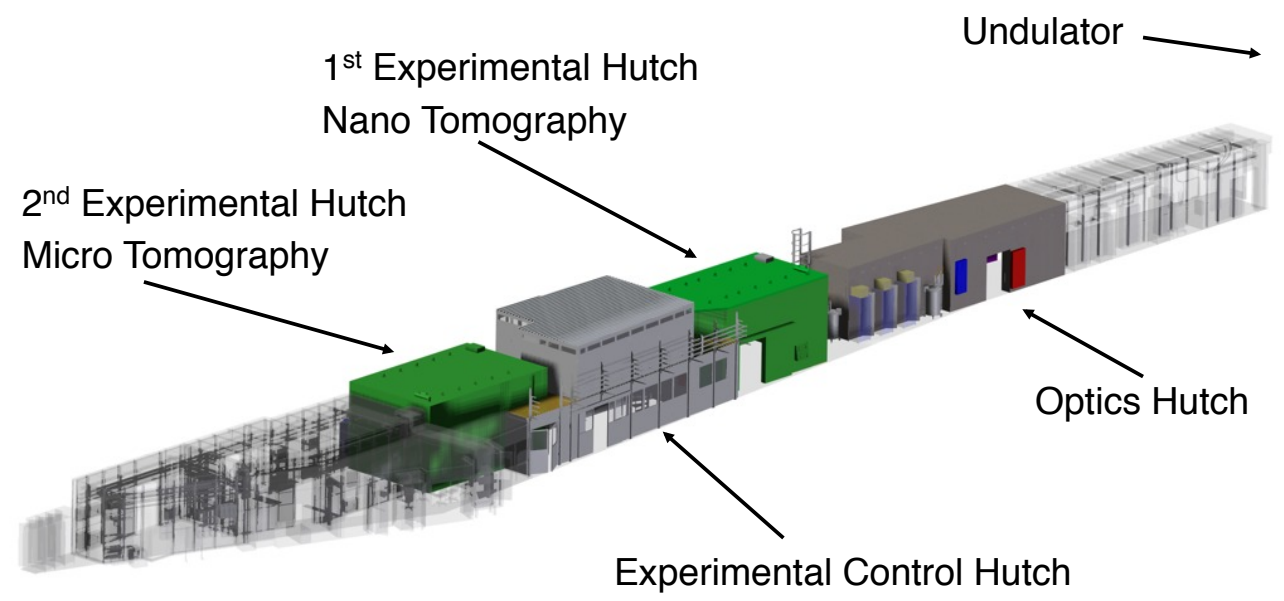

Figure 1. Overview of Imaging Beamline IBL / P05 at PETRA III.

\section{THE IMAGING BEAMLINE P05}

The imaging beamline IBL (P05) at the $3^{\text {rd }}$ generation storage ring PETRA III has a micro tomography and a nano tomography end station, ${ }^{1}$ as shown in the schematic layout in figure 1 . With an energy range of 5 to 50 $\mathrm{keV}$ the beamline is suitable for low absorbing biological and medical applications as well as higher absorbing engineering materials. As an insertion device a $2 \mathrm{~m}$ undulator is used providing $\mathrm{x}$-rays for the imaging beamline P05. The P05 beamline is set up on a shared sector with the micro- and nano-probe beamline P06 using two undulators canted by $5 \mu \mathrm{rad}$. These two beamlines are working completely independent of each other. Due to the way the two beamlines are sharing the sector the overall length of IBL is $90 \mathrm{~m}$. Currently the optics hutch is equipped with a double crystal monochromator (DCM) with silicon single crystals (111 and 311) designed by DESY and built by Oxford Instruments. This DCM is ideal for tomographic experiments needing high monochromatization, like for example absorption edge tomography $\left(\Delta E / E \approx 10^{-4}\right)$. In oder to increase the flux e.g. for fast time resolved experiments a double multilayer monochromator (DMM) will be installed. The installed multilayer crystals will allow an energy bandwidth of $\Delta E / E \approx 10^{-2}$ with multilayer coatings made in house by HZG. ${ }^{1,2}$ Both monochromators are cooled independently using liquid nitrogen. Furthermore, a large vacuum tank is set up at the end of the optics hutch giving enough space for additional apertures and $\mathrm{x}$-ray optical components like e.g. mirrors or CRLs for the cone beam setup in the nano tomography station. The micro tomography station of IBL is located at the end of the beamline, at $87.5 \mathrm{~m}$ in order to reach the optimal requirements needed for micro tomography.

\subsection{Micro Tomography Setup}

The micro tomography experimental hutch has a length of $9 \mathrm{~m}$ and houses the micro tomography experiment providing additional space for extended sample environments or stand alone in situ experiments. Due to the sample-to-source distance the field of view (FOV) is sufficient to investigate samples of several millimetres in diameter (see table1). With the setup spatial resolutions in the micrometer range and below can be achieved which covers a wide range of applications in material sciences as well as in biology or medicine. The experimental setup is outlined in figure 2. The massive granite substructure is needed to assure high mechanical stability for the components, which is necessary in order to achieve such spatial resolutions. On top of this substructure the camera tower is mounted on another granite, decoupled from the sample stage. The sample rotation and translation as well as the camera translation in beam direction are run by high precision air bearing stages mounted on the granite substructure. The whole granite substructure, the camera on its granite tripod as well as the sample stage are adjustable in pitch, yaw, roll and height independently of each other. Additionally the granite substructure can be moved horizontal to the beam direction. 


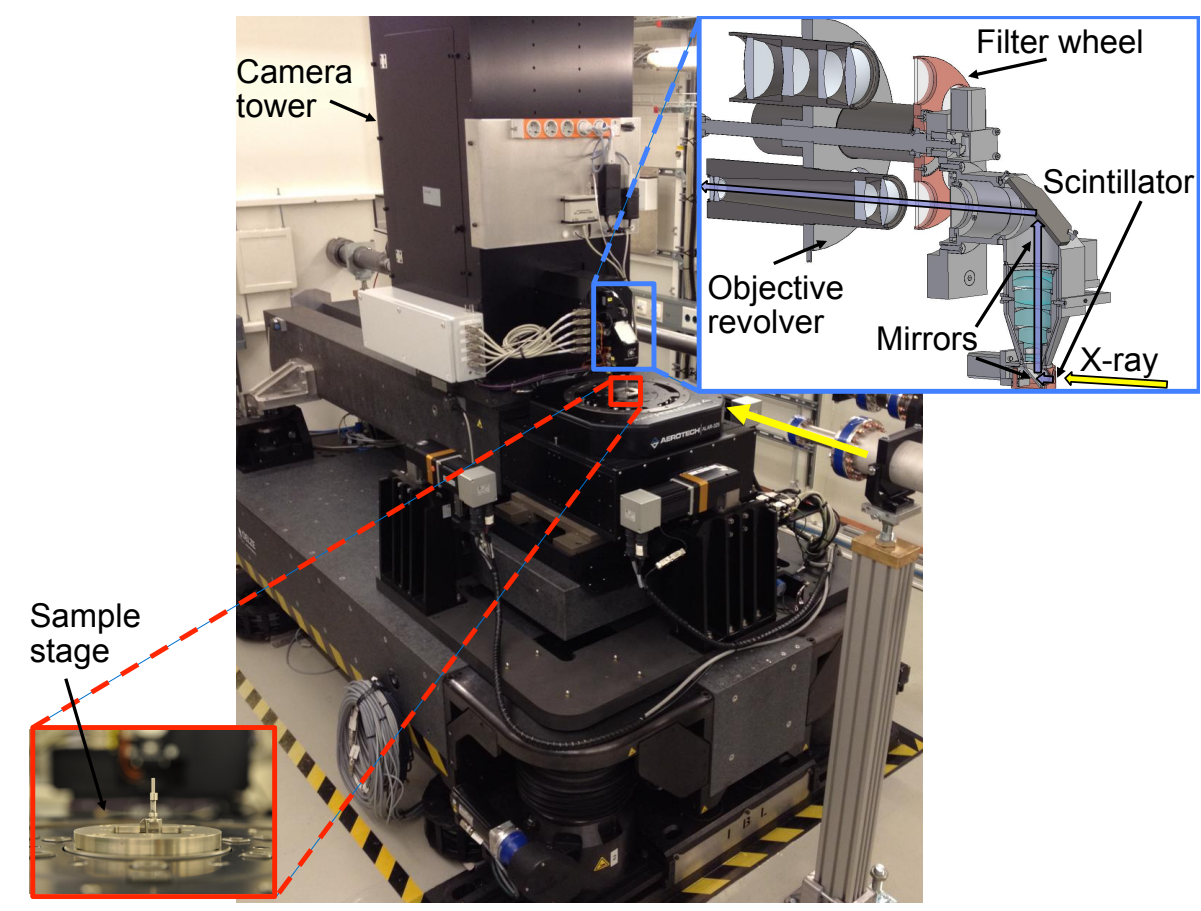

Figure 2. The micro tomography setup at the imaging beamline IBL / P05 at PETRA III.

Currently a $\mathrm{CdWO}_{4}$ crystal is used as a scintillator mounted in front of the optics of the camera tower (see figure 2 , top right inset). Three mirrors are guiding the visible light through a set of lenses, the chosen objective lens $(5 \mathrm{x}-40 \mathrm{x})$, which are mounted on the objective revolver and finally up into the CCD camera, which is mounted inside the camera tower. The optics are calculated for a chip of the size of $60 \mathrm{~mm}^{2}$, the currently installed CCD camera has a chip of $3056 \times 3056$ with a pixel size of $12 \mu \mathrm{m}$. Two cameras can be installed in the camera tower and a mirror allows switching between them. Additionally, a filter wheel is installed in the setup as well as an optical shutter. An x-ray shutter is installed directly behind the slit system and consists of an $3 \mathrm{~mm}$ thick tungsten plate minimising radiation damage.

In order to check for higher harmonics, a liquid nitrogen cooled fluorescence detector is installed perpendicular to the $\mathrm{x}$-ray beam. Higher harmonics are subsequently suppressed by detuning the second Si crystal in the DCM. Acquisition times of a tomographic scan are currently rather high (around 1 hour depending on the selected magnification) due to the choice of the camera and the necessity to take multiple reference images because of beam instabilities. Before the data reconstruction procedure can be started the reference images are correlated with the projections of the sample in order to find the best match. This procedure assures a high contrast reconstruction with good spatial resolution. The reconstruction itself is based on the filtered back projection method and the user interface and data pre-processing is written in IDL.

Table 1. P05 beamline parameters

\begin{tabular}{|c|c|c|c|c|}
\hline Magnification & $5 \mathrm{x}$ & $10 \mathrm{x}$ & $20 \mathrm{x}$ & $40 \mathrm{x}$ \\
\hline FOV $[\mathrm{mm}]$ & $7.4 \times 1.9$ & $3.8 \times 1.9$ & $1.9 \times 1.9$ & $0.9 \times 0.9$ \\
\hline Resolution $[\mu \mathrm{m}]$ & 3 & 1.5 & $<1$ & $<1$ \\
\hline
\end{tabular}

Since 2013 the micro tomography station is in full user operation in the absorption mode. In autumn last year a fully automated (unattended) sample changer robot arm (Universal Robots UR2) was installed at the micro tomography setup. The fitting and integration into the setup was done in-house by the HZG technical department. Up to 20 samples can be installed in the holder and are distinguish by a data matrix. 


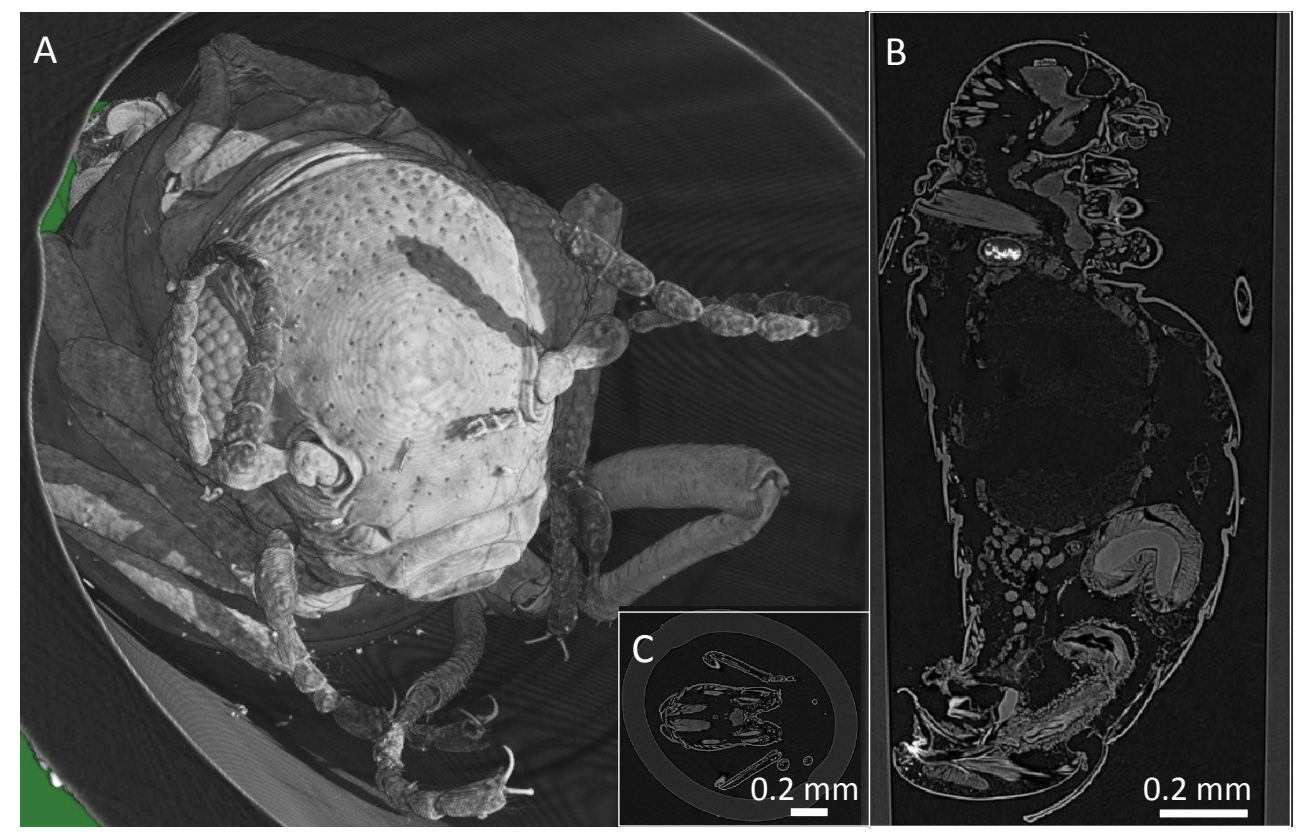

Figure 3. Volume rendering of a moss flea (Caurinus sp., A) and two reconstructed slices of the insect (B, C).

The software for controlling the robot and the sample changing procedure are implemented into tango. As mentioned above, the available space at the micro tomography setup allows for extended sample environments. For example an in situ furnace was designed specifically for P05 $\mu \mathrm{CT}$ setup at IBL. The design and construction was conducted by HZG technical department. The oven can heat up to $800^{\circ} \mathrm{C}$ and is equipped with a gas inlet and outlet.

\subsubsection{First Results}

The imaging beamline IBL/P05 is dedicated to material sciences covering an energy range from 5 to $50 \mathrm{keV}$. Therefore, it is as well highly suited for the study of soft bio-materials and classical materials science samples, like sclerotized insect cuticle as well as bone or metal alloys. In the following some examples will be presented, acquired during the first year of user operation.

$\mathrm{SR} \mu \mathrm{CT}$ allows to acquire the anatomy of insects at sub micron spatial resolution instead of using histology which can take up to a month for a single insect. A tomogram of a freeze dried moss flea (Caurinus sp.) was acquired at the imaging beamline IBL. This tiny insect species is crucial to reconstruct the evolution of the whole order. A detailed understanding of phylogenetic relationships is crucial for biologist in the context of understanding evolution and fundamental concepts of functional morphological and physiological processes. Also in an applied context of bio-materials and bio-inspired materials a thorough understanding of relationships among species is important to stimulate the discovery of new interesting candidate species. The insects investigated in this study are of a size of about $2 \mathrm{~mm}$ in length and $1 \mathrm{~mm}$ in diameter and were measured at a photon energy of $8 \mathrm{keV}$ at a $20 \mathrm{x}$ magnification, resulting in a pixel size of $1.22 \mu \mathrm{m}$.

The reconstructed volume is $1.25 \times 1.14 \times 2.33 \mathrm{~mm}^{3}$. Most of its anatomy (e.g. muscles, skeleton and nerves) was completely unknown and is now documented for the first time using high-resolution $\mathrm{SR} \mu \mathrm{CT}$ (see figure 3). The anatomy of a high number of related species has to be studied in order to reconstruct the ancient pattern of the last common ancestor and the relationships of the species in question to address in an broader context the evolution of insects. This can be accomplished in a highly efficient way compared to classical established techniques in biology only by the use of high-quality $\mathrm{SR} \mu \mathrm{CT}$ data at the imaging beamline IBL. The exceptional high density resolution of the data allows for a fast and accurate anatomical analysis. 

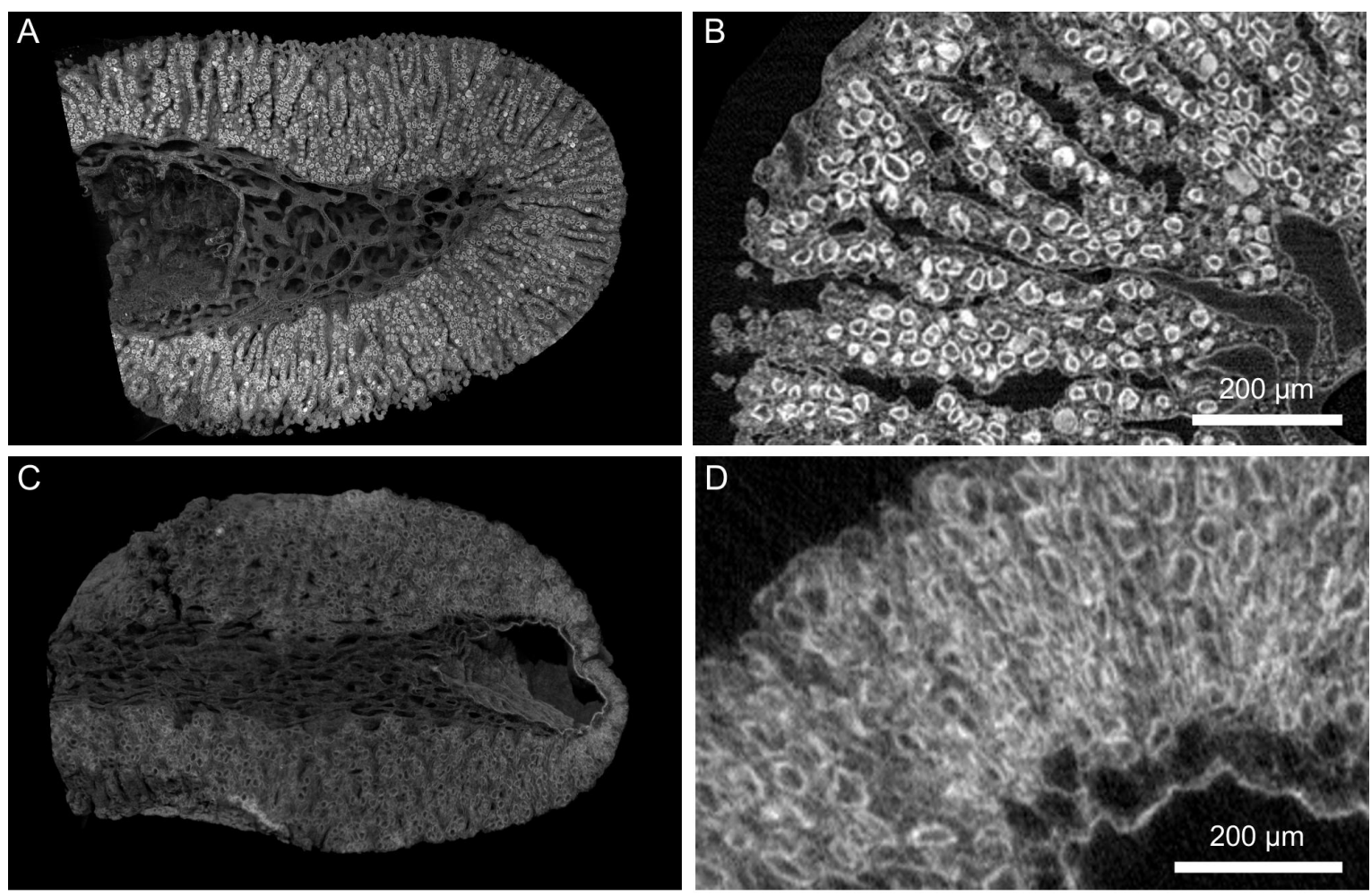

Figure 4. Volume renderings virtually half sectioned (A,C) and image planes (B,D) of O. lobularis imaged in an expanded state at beamlines P05 (A,B) and BW2 (C,D).

Another example from biology requires a higher photon energy. Sponges (Porifera) are sessile, filter-feeding mainly marine animals. In recent years, interest in sponges raised due to their high diversity of secondary metabolites of pharmaceutical value. ${ }^{3}$ Therefore, sponges became interesting for pharmacology and biotechnology, aiming the production of sponge biomass and metabolites. Soon it became evident that a more detailed knowledge on the general biology of sponges is a prerequisite for successful cultivation. On the other hand, sponges are highly interesting to understand the early evolution of animals since they are thought to have evolved first among all extant animal groups. The simplicity of the sponge body with its characteristic canal system which serves as a filtering unit to gain nutrients and oxygen as well as to expel metabolic waste products is partly primordial and partly derived. In recent years it became more and more evident that sponges are especially informative on the evolution of the nervous system and of muscle cells. ${ }^{4,5}$ Although sponges lack nerve and muscle cells, they are contractile and able to respond specifically upon external stimuli and display endogenous rhythms (for a review see Nickel et.al. ${ }^{6}$ ).

It has been demonstrated that the sponge epithelium is the contractile tissue in sponges. ${ }^{6}$ The phasic contraction of freshwater sponges is a challenge to this hypothesis and $\mathrm{SR} \mu \mathrm{CT}$ at the sub-micrometer resolution will allow for evaluating this particular hypothesis and its alternative hypothesis, the contractility of the sponge mesenchyme (connective tissue). Aiming to test the alternative hypothesis $\mathrm{x}$-ray tomograms were acquired at the BW2 beamline $^{7}$ at the former storage ring DORIS III at DESY (shut down in 2012). In order to measure Oscarella lobularis samples of about $3 \mathrm{~mm}$ in diameter a photon energy of $14 \mathrm{keV}$ with an effective voxel size of $2.94 \mu \mathrm{m}$ was used. The samples were fixed in contracted and expanded states in a solution of $2.5 \%$ glutharaldehyde and contrasted using $1 \% \mathrm{OsO}_{4}$. Specimens were critical point dried and glued to interchangeable sample holders. In 2013 we acquired first imaging results of a similar Oscarella lobularis sample at the new imaging beamline P05. A direct comparison of the different data sets measured at the two beamlines suggested that the high spatial and density resolution required to answer the hypothesis formulated for the role of mesenchyme contraction could be reached with the new P05 setup. 

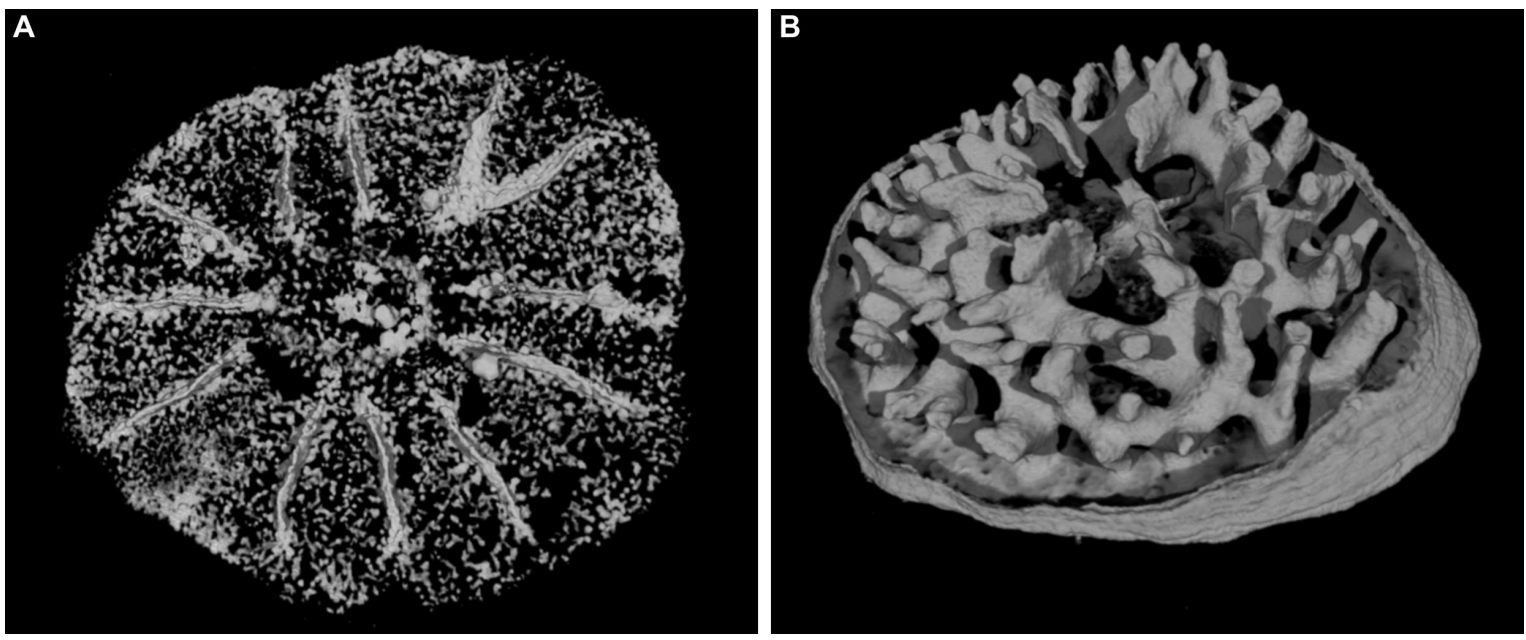

Figure 5. Volume renderings of coral larvae skeleton. Two different developmental stages are shown 2 days (A) and 28 days (B) after settling.

At the beamline P05 the sample was measured at $15 \mathrm{keV}$ resulting in an effective voxel size of $2.52 \mu \mathrm{m}$. More experiments at higher magnification are planned after the extension phase of PETRA III. The quantitative and qualitative analysis of the obtained high resolution 3D reconstruction datasets was done using custom developed macro sets for ImageJ (Fiji) and VG StudioMax.

$\mathrm{SR} \mu \mathrm{CT}$ is also a vital tool to study the mechanism and aspects of biomineralization. The world ocean is the largest sink of anthropogenic $\mathrm{CO}_{2}$ and heat. As $\mathrm{CO}_{2}$ diffuses in seawater, it behaves like a weak acid leading to a decrease in $\mathrm{pH}$. This process is referred to as 'Ocean Acidification', and results in acidification of marine waters on a global scale. The impacts of increased $\mathrm{CO}_{2}$ on scleractinian corals may include not only reduced calcification, but may ultimately include dissolution of calcified skeletons. ${ }^{8,9}$ An international research group lead by Jaap Kaandorp (Universiteit van Amsterdam) studied aspects of biomineralization in the context of $\mathrm{CO}_{2}$ driven ocean acidification (OA) and climate change by analyzing biomineralization in the skeletal development of the coral Acropora millepora by means of $\mathrm{SR} \mu \mathrm{CT}$ at the imaging beamline IBL (see figure 5). The sample had a size of $3 \mathrm{~mm} \times 7 \mathrm{~mm}$ and was measured at an energy of $20 \mathrm{keV}$ using an effective pixel size of $2.5 \mu \mathrm{m}$.

\subsection{Nano Tomography Setup}

The nano-tomography experiment is in the late commissioning phase. The experimental hutch has a length of $9 \mathrm{~m}$ and is situated just behind the optics hutch at a distance of $63-72 \mathrm{~m}$ from the source. ${ }^{10}$ Though the smaller source distance impedes $\mathrm{x}$-ray focussing with large source demagnification, the proximity to the optics hutch makes it possible to create a virtual source situated about $4 \mathrm{~m}$ in front of the experimental hutch. Furthermore, the smaller beam cross-section allows a higher photon flux in the $\mathrm{x}$-ray microscopy mode. The complete experiment is mounted on a $6.8 \mathrm{~m}$ long granite optical bench. Four air-bearing granite sliders are used to position the experimental components with respect to each other. Each slider can move the complete length of the optical bench, only limited by the other sliders. Of the four sliders, one is used for the detector, the sample stage is mounted on another one and two sliders are outfitted with six-axes kinematics for positioning of $\mathrm{x}$-ray optics. This versatile setup makes an excellent basis for $\mathrm{x}$-ray full field microscopy and also for tomography experiments.

\subsubsection{First Results}

An x-ray full field microscope has been realized at the nano tomography end-station, using a compound refractive lens (CRL) as objective lens and a rolled prism lens as a condenser optics. The overall length of the setup is about $5 \mathrm{~m}$ using these optics. Tests have been performed at energies of 17.4 and $30 \mathrm{keV}$ and $\mathrm{x}$-ray magnifications of about 25 have been achieved. A $11 \mathrm{~m}$ thick LSO scintillator has been used in the detector system. 


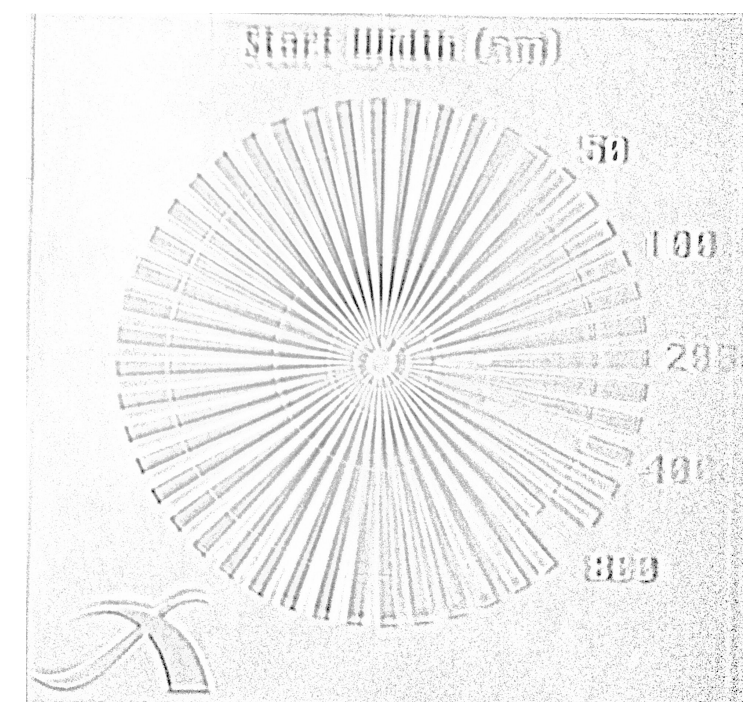

Figure 6 . Image of a test pattern acquired at $17.4 \mathrm{keV}$ at the nano tomography beamline at IBL. The inner circle are the $50 \mathrm{~nm}$ lines, followed by the $100 \mathrm{~nm}$ lines.

The microscope objectives yielded an additional magnification of $\mathrm{M}=10$ or $\mathrm{M}=20$ and a $\mathrm{PCO} 4000 \mathrm{CCD}$ camera has been installed for image acquisition. At an photon energy of $30 \mathrm{keV}$, a spatial resolution better than $260 \mathrm{~nm}$ (line and space) over the entire field of view of $80 \times 80 \mu \mathrm{m}$ has been achieved. ${ }^{11}$ At $\mathrm{E}=17.4 \mathrm{keV}$, a field of view of $50 \times 50 \mu \mathrm{m}^{2}$ and a resolution of $180 \mathrm{~nm}$ line and space was reached, i.e. structures with a width of $90 \mathrm{~nm}$ could be resolved (compare figure 6).

\section{CONCLUSIONS}

The imaging beamline P05/IBL at PETRA III is well suited for a wide range of applications, from standard attenuation based tomography in the micrometer range, over phase contrast methods all the way to $\mathrm{x}$-ray microscopy in the nanometer range. The available energies ranging from 5 up to $50 \mathrm{keV}$ cover a broad range of research fields from biological to metal samples. Thanks to the large space available at the experimental setup of the micro tomography, extended sample environments can be installed, allowing for in situ experiments. During the first year in full user operation the micro tomography station has proven its high potential. In comparison to the W2 and BW2 beam lines at the DORIS III storage ring the micro tomography at IBL has already proven a much higher spatial resolution. At the nano tomography setup a full field $\mathrm{x}$-ray microscope has been installed and tested using CRLs and rolled prism lenses. It is optimised for energies of 17.4 and $30 \mathrm{keV}$ and resolutions of 180 and $260 \mathrm{~nm}$ have been achieved. Additional space in the monochromator tank allows for other optic concepts and setups, which will be installed in the future.

\section{ACKNOWLEDGMENTS}

I. Greving acknowledges the Virtual Institute of New X-ray Analytic Methods in Material Science (VI-NXMM) for funding. M.O. gratefully acknowledges financial support from the German Research Foundation (DFG) via SFB $986 \mathrm{M}^{3}$, project Z2. The authors thank Felix Marschall, Harald Vogt and Arndt Last of the Karlsruhe Institute for Microstructures (IMT) and the Karlsruhe Institute for Nano Micro Facility (KNMF) for the design and fabrication of the lenses. J.U.H. acknowledges the providence of samples and scan data for this publication from Jaap A. Kaandorp (Universiteit van Amsterdam) and Michael Nickel (Bionic Consulting). 


\section{REFERENCES}

1. A. Haibel, F. Beckmann, T. Dose, J. Herzen, M. Ogurreck, M. Müller, and A. Schreyer, "Latest developments in microtomography and nano tomography at PETRA III," Powder Diffraction 25(02), pp. 161-164, 2010.

2. M. Störmer, C. Horstmann, O. Häussler, E. Spiecker, F. Siewert, F. Scholze, F. Hertlein, W. Jäger, and R. Bormann, "Mono- and multi- layer mirrors for current and next-generation light sources," SPIE 7077, p. $707705,2008$.

3. N. Fusetani, Drugs from the sea, Karger, Basel, 2000.

4. P. Steinmetz, J. Kraus, C. Larroux, J. Hammel, A. Amon-Hassenzahl, E. Houliston, G. Worheide, M. Nickel, B. Degnan, and U. Technau, "Independent evolution of striated muscles in cnidarians and bilaterians," Nature 487, p. 231, 2012.

5. M. Nickel, "Evolutionary emergence of synaptic nervous systems: what can we learn from the non-synaptic, nerveless porifera," Invertebrate Biology 129, pp. 1-16, 2010.

6. M. Nickel, C. Scheer, J. Hammel, J. Herzen, and F. Beckmann, "The contractile sponge epithelium sensu lato-body contraction of the demosponge Tethya wilhelma is mediated by the pinacoderm," J Exp Biol 214, pp. 1692-1698, 2011.

7. F. Beckmann, U. Bonse, and T. Biermann, "New developments in attenutation and phase-contrast microtomography using synchrotron radiation with low and high photon energies," Proc. of SPIE 3772, pp. 179-187, 1999.

8. J. Orr, V. Fabry, O. Aumont, L. Bopp, and S. Doney, "Anthropogenic ocean acidification over the twentyfirst century and its impact on calcifying organisms," Nature 437, pp. 681-686, 2005.

9. J. Kleypas, R. Feely, V. Fabry, and C. Langdon, Impacts of ocean acidification on coral reefs and other marine calcifiers: a guide for future research, NSF, NOAA, U.S. Geological Survey, St. Petersburg, 2006.

10. M. Ogurreck, F. Wilde, J. Herzen, F. Beckmann, V. Nazmov, J. Mohr, A. Haibel, M. Müller, and A. Schreyer, "The nano tomography endstation at the PETRA III imaging beamline," Journal of Physics: Conference Series 425, p. 182002, 2013.

11. F. Marschall, A. Last, M. Simon, M. Kluge, V. Nazmov, H. Vogt, M. Ogurreck, I. Greving, and J. Mohr, "X-ray full field microscopy at $30 \mathrm{keV,"} \mathrm{Journal} \mathrm{of} \mathrm{Physics:} \mathrm{Conference} \mathrm{Series} \mathrm{499,} \mathrm{p.} \mathrm{012007,} 2014$. 